\title{
Comparison of existing methods for the calculation of the infinite water depth free-surface Green function for the wave-structure interaction problem
}

\author{
Chunmei Xie, Youngmyung Choi, François Rongère \\ Alain H. Clément, Gérard Delhommeau, Aurélien Babarit* \\ Ecole Centrale de Nantes, 1 Rue de la Nö̈, 44300 Nantes, France
}

\begin{abstract}
In this study, the mathematical expressions and numerical methods for the free-surface Green function of the linearized wave-structure problem in deep water and in the frequency domain are investigated. Twelve different expressions are reviewed and analyzed. All these expressions are exact mathematical solutions for the propagation of waves from a pulsating source located in the fluid domain. However, their numerical evaluation is challenging. Dedicated numerical methods have been developed. They include series expansions, polynomials, table interpolations, multipole expansions, approximations with elementary functions, etc. In this work, four methods were implemented: the Newman's method [1], the Delhommeau's method [2], the Telste-Noblesse's method [3] and the Wu et al.'s method [4]. Their CPU time and accuracy are compared. It is found that the average computational time for Newman's method is $5.745 \times 10^{-7}$. It is $5.782 \times 10^{-8}$ for the Delhommeau's method. For Telste-Noblesse's method and $\mathrm{Wu}$ et al.'s methods, they are $4.642 \times 10^{-8}$ and $1.491 \times 10^{-9}$, respectively. The accuracy is respectively $6 \mathrm{D}(6$ decimals $), 5 \mathrm{D}$ and $3 \mathrm{D}$ for the Newman's method, the Telste-Noblesse's method and the Wu et al.'s method. For the Delhommeau's method, it is 3D except when the vertical coordinate is close to 0 . The accuracy of the Delhommeau's method can be increased significantly by
\end{abstract}

\footnotetext{
${ }^{*}$ Corresponding author

Email address: aurelien.babarit@ec-nantes.fr (Aurélien Babarit)
} 
refining the discretization of the space variables for the tabulated functions and by using higher interpolation methods, at cost of increased computational time. Keywords: Wave-structure interaction, linear potential theory, Green function, Numerical modeling

\section{Introduction}

The diffraction and radiation of water waves by floating bodies with zero mean forward speed and in deep water are phenomena of uttermost importance in ocean engineering. They must be taken into account in the design

5 of marine structures such as oil and gas platforms or marine renewable energy converters. In the industry, boundary element methods (BEM)-based codes are usually used to calculate these effects and determine the pressure fields and wave-induced forces acting on the structures. BEM codes rely on the linear free surface potential flow theory which itself relies on the free-surface Green function and its derivatives.

Two distinct numerical problems must be overcome in the implementation of BEM codes [1. Firstly, the discretization of the body surface by a large number of panels leads to the construction of a dense linear system of equations which must be solved by suitable algorithms. Secondly, the evaluation of 15 the Green function and its derivatives is challenging because of their singular behaviour at the origin. Typically, the numerical complexity of BEM codes is proportional to $O\left(N^{2}\right)$ or $O\left(N^{3}\right)$ with $N$ the number of unknowns. The Green function is computed $O\left(N^{2}\right)$ times to set up the linear system. The linear system is solved either by an iterative method with $O\left(N^{2}\right)$ complexity or by Gauss 20 elimination with $O\left(N^{3}\right)$ complexity. The complexity can be reduced down to $O(N \log N)$ using acceleration algorithms such as the precorrected Fast Fourier Transform(pFFT) [5]. Although the computational time for the evaluation of the Green function and its derivatives is not fully representative of the computational time needed by a BEM code to evaluate the diffraction and radication 25 by a floating body, it is still considered as one of the challenges for efficient 
three-dimensional computation of hydrodynamic coefficients and forces.

In the frequency domain, this Green function is solution of the following boundary value problem:

$$
\begin{aligned}
& \nabla^{2} G_{\infty}=\delta\left(x_{M}-x_{P}\right) \delta\left(y_{M}-y_{P}\right) \delta\left(z_{M}-z_{P}\right) \quad \text { in } \quad z<0 \\
& G_{\infty z}-\omega^{2} / g G_{\infty}=0 \quad \text { on } \quad z=0 \\
& G_{\infty}=O(1 / \sqrt{R}) \quad \text { as } \quad R \rightarrow \infty
\end{aligned}
$$

where $G_{\infty}$ is the Green function in deep water, $\delta$ is the Dirac function, $R$

30 is the distance between the source point $M\left(x_{M}, y_{M}, z_{M}\right)$ and the field point $P\left(x_{P}, y_{P}, z_{P}\right) . \omega$ is the wave frequency and $g$ is the gravity. In Eq. 1 1 , the first equation is the Laplace equation; the second equation derives from the linearized free-surface conditions and the last equation is the radiation condition at infinity.

This boundary value problem was studied extensively during the 1940s and 1950s and exact mathematical expressions for its solution were obtained and those expressions were reviewed by Wehausen and Laitone [6]. Later, Ursell [7] developed an expression involving a series of spherical harmonics for a heaving semi-sphere. A modified Green function from Haskind's representation was then proposed by Kim [8]. It was implicitly provided by Havelock in [9]. This modified expression was also re-derived by Hearn [10. Finally, an integral representation in terms of an exponential integral was introduced independently by Guevel [1], Martin [12] and Noblesse [13].

The numerical evaluation of these expressions (and the evaluation of their derivatives) is challenging because of the complexity of the involved mathematical expressions, the mathematical singularity in $M=P$ and the associated computational time [1] [3. This is an issue for BEM codes because the Green function (and its derivatives) must be evaluated many times to solve the wave-structure interaction problem for each considered frequency. Thus, with the rapid development of computing resources, the focus shifted in the 1980s so from the derivation of mathematical expressions for the Green function towards the algorithms and numerical methods for its efficient numerical computation. Noblesse [13] 14 proposed two complementary near-field and far-field single 
integral representations in terms of the exponential integral. An asymptotic expansion and convergent ascending series are used to calculate the Green function for large and small distances between the source and field point, respectively. Two complementary Taylor series expansions are also provided when the nondimensional spatial coordinates approach zero. In 1986, Telste and Noblesse [3] published a numerical code for the evaluation of the Green function and its derivatives based on Noblesse's previous study 14. The computational domain was divided into five sub-domains.

In 1984, Newman [15] used the Romberg quadrature to evaluate the finite integral of the free-surface Green function with double precision accuracy. In the same year, he proposed a new series expansion [16]. It is computationally efficient at small and moderate radial distances between the source and field points.

${ }_{65}$ A year later, he [1] proposed an algorithm for the evaluation of Green function and its derivatives in infinite and constant finite depth. The computational domain is divided into several domains for which polynomial approximations are provided including the series expansion from his previous paper 16. This algorithm is implemented as a standard subroutine named as "FINGREEN" in the boundary element method (BEM)-based code WAMIT [1, 17].

In 1991, a new algorithm based on a polynomial series approximation was proposed by Chen et al. [18, 19, 20]. Double Chebyshev polynomials approximations with special functions are used to evaluate the Green function for infinite and finite water depth. Chen's algorithm is implemented in the BEM code HYDROSTAR. A similar algorithm with double Chebyshev polynomials is also proposed by Wang in 21.

To reduce the computational time, Delhommeau developed a technique involving four tabulated functions and Lagrange interpolations. This technique is implemented in the BEM code AQUADYN [2] and in the open source code 80 NEMOH [22].

Other expressions and numerical methods are available. In a number of research works, the Green function is decomposed into three parts: a free-space singularity, a non-oscillatory local flow and waves. Ba et al. 23 . and Ponizy et 
al. 24] provided approximation methods for the non-oscillatory local flow term based on the use of a coordinates-transformation and a function-transformation. Proper coordinates and function transformations reduce the problem of approximating singular functions for unbounded domains into that of approximating smoothly varying functions over finite domains. Linear table interpolation can thus be used. Peter and Meylan proposed an eigenfunction expansion representation [25]. In 2011, a semi-analytical method was developed with the Haskind-Havelock kernel calculated by a singularity subtractive technique [26]. The same year, the multipole expansion was extended for the infinite water depth free surface Green function [27].

Recently, Wu et al. 4] proposed a global approximation of the Green function 95 and its derivatives based on Noblesse's paper [14, 3]. A simple approximation involving elementary functions is given for the local flow component. It does not require dividing the computational domain into multiple sub-domains.

A completely different approach was proposed by Clément [28]. It is based on a second order differential equations for the Green function. However, a challenge with this method is its initialization.

It should be noted that the numerical errors associated to BEM-based codes stem from several sources as discussed in [4. They include the approximation of the body surface by a large number of panels; the approximation of the variations (piecewise constant, linear, quadratic, or higher-order) of the densities of the singularities (source, dipole) distributions over the surface panels; the numerical integration of the Green function and its gradient over the panels; and the numerical evaluation of the Green function and its gradient. Thus, the effect of numerical errors in the numerical evaluation of the Green function and its derivatives for practical computations is difficult to estimate. Liang et al. [29] investigated the accuracy of linear and second order wave loads for a hemisphere and a freely Floating Production Storage and Offloading (FPSO) unit. They used the $\mathrm{Wu}$ et al.'s method. They showed that using this 3D accuracy method for the Green function and its gradient doesn't make much difference for the hydrodynamic coefficients and forces when compared to results obtained using 

practical cases.

In this paper, various expressions for the free-surface Green function are reviewed and presented. Several numerical methods have been proposed among which four methods were selected and compared with respect to accuracy and computational time. The two first ones are the Newman's and Telste-Noblesse's methods. They were selected because they are widely known in the industry and academia. The Newman's method is implemented in the industry standard BEM code WAMIT. The Delhommeau's method was selected because it is implemented in the open source code NEMOH. The fourth method is that of $\mathrm{Wu}$ et al.. It was selected because it provides a global approximation which is easy to implement and doesn't need subdivisions. Thus it may be more suitable for parallel computation.

\section{Mathematical expressions for the free-surface Green function in deep water}

130

In this study, the time factor of the complex potential is taken as $e^{-i \omega t}$. The coordinates and variables are depicted in Fig. 1. The mean free surface level is located at the plane $z=0$. The vertical axis $z$ points upwards. The source point $P\left(x_{P}, y_{P}, z_{P}\right)$ and the field point $M\left(x_{M}, y_{M}, z_{M}\right)$ are both lying on or under the free surface $\left(z_{P} \leq 0, z_{M} \leq 0\right)$. The image source point $P^{\prime}\left(x_{P}, y_{P},-z_{P}\right)$ is the mirror of the source point $P$ with respect to the mean free surface. The horizontal distance between the source point $P$ and the field point $M$ is denoted by $r$. The vertical distance between the image source point $P^{\prime}$ and the field point $M$ is $-Z$. The distance between the source and field points is denoted by $R$ and the distance between the image source point and the field point is ${ }_{140} R_{1}=\sqrt{r^{2}+Z^{2}}$. The angle $\theta$ is defined by $\cos \theta=-Z / R_{1}$. The relations 
between the coordinates are given by:

$$
\begin{aligned}
& r=\sqrt{\left(x_{M}-x_{P}\right)^{2}+\left(y_{M}-y_{P}\right)^{2}} \\
& Z=z_{M}+z_{P} \\
& R=\sqrt{\left(x_{M}-x_{P}\right)^{2}+\left(y_{M}-y_{P}\right)^{2}+\left(z_{M}-z_{P}\right)^{2}}
\end{aligned}
$$

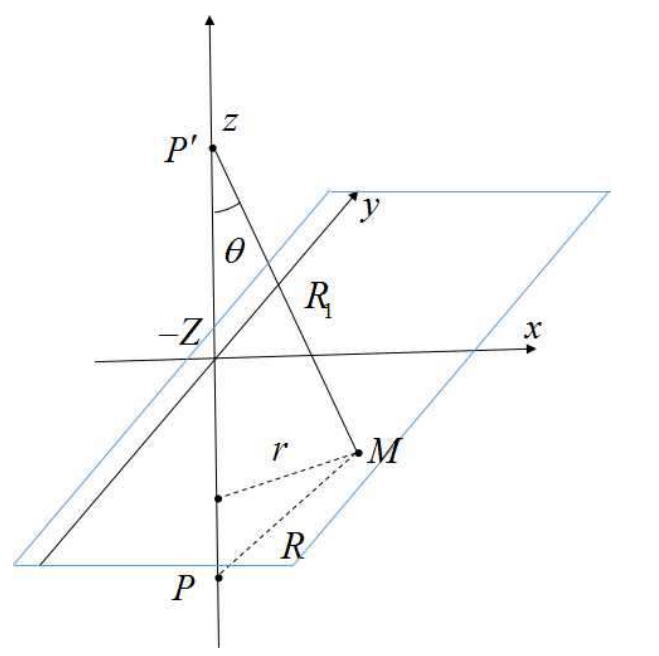

Figure 1: source and field point

\subsection{Expressions of the first type}

We define the expressions of the Green function of the first type as the expressions that can be written as:

$$
-4 \pi G_{\infty}(r, Z, \omega)=\frac{1}{R}-\frac{1}{R_{1}}+g_{-}(r, Z, \omega)+2 i \pi k_{0} e^{k_{0} Z} J_{0}\left(k_{0} r\right)
$$

$g_{-}(r, Z, \omega)$ is called the free-surface term whereas the first two terms are the Rankine source $(1 / R)$ and the image source $\left(1 / R_{1}\right)$ contributions, respectively. One can note that these two source distributions cancel each other when the field point is located on the mean free surface $z=0 . k_{0}$ is the wave number defined by $k_{0}=\omega^{2} / g$ and $J_{0}\left(k_{0} r\right)$ is the zeroth order Bessel function of the first kind. 


\subsubsection{Expression 1}

The classical expression for the free-surface term reads [28:

$$
g_{-}(r, Z, \omega)=2 P V \int_{0}^{\infty} \frac{k}{k-k_{0}} e^{k Z} J_{0}(k r) d k
$$

where $P V \int$ represents the Cauchy principal integral. For $\omega=0$ and $\omega \rightarrow \infty$, one can show that:

$$
\left\{\begin{array}{l}
g_{-}(r, Z, 0)=\frac{2}{R_{1}} \\
g_{-}(r, Z,+\infty)=0
\end{array}\right.
$$

\subsubsection{Expression 2}

The expression used in the BEM codes NEMOH and AQUADYN [2, 22] is:

$$
g_{-}(r, Z, \omega)=\frac{2 k_{0}}{\pi} \mathbf{R e}\left[\int_{-\frac{\pi}{2}}^{\frac{\pi}{2}}\left[\mathbf{J}(\zeta)-\frac{1}{\zeta}\right] d \theta\right]
$$

where $\zeta=k_{0}(Z+i r \cos \theta)$ and $\mathbf{J}(\zeta)=e^{\zeta}\left[\mathbf{E}_{1}(\zeta)+i \pi\right]$. $\mathbf{E}_{1}(\zeta)$ is the complex exponential function. In this expression, the free-surface term only involves a finite integral of the complex exponential integral whereas it is an infinite integral in the classical expression 4. Therefore, a direct numerical integration may be used to evaluate it. However, it should be noted that the integration kernel tends to infinity with a logarithmic behavior when both $r$ and $Z$ tend to 0.

Note that in the BEM codes NEMOH and AQUADYN, the evaluation of the Bessel function in the imaginary part of the Green function is replaced by the evaluation of the following expression:

$$
\operatorname{Im}\left[-4 \pi G_{\infty}(r, Z, \omega)\right]=2 k_{0} \operatorname{Re}\left[\int_{-\frac{\pi}{2}}^{\frac{\pi}{2}} e^{\zeta} d \theta\right]
$$

\subsection{Second type}

We define the expressions of the Green function of the second type as:

$$
-4 \pi G_{\infty}(r, Z, \omega)=\frac{1}{R}+g_{0}(r, Z, \omega)+2 i \pi k_{0} e^{k_{0} Z} J_{0}\left(k_{0} r\right)
$$

170

By comparing to the expressions of the first type, one can see that the contribution of the image source is included in the free-surface term $g_{0}(r, Z, \omega)$. 


\subsubsection{Expression 3}

The free-surface term $g_{0}$ is given in Wehausen and Laitone's book [6], equation $\left(13.17^{\prime \prime}\right)$. It reads:

$$
g_{0}(r, Z, \omega)=P V \int_{0}^{\infty} \frac{k+k_{0}}{k-k_{0}} e^{k Z} J_{0}(k r) d k
$$

It can be shown that it is equivalent to expression 1 by using the Lipschitz's integral: $1 / R_{1}=1 / \sqrt{r^{2}+Z^{2}}=\int_{0}^{\infty} e^{k Z} J_{0}(k r) d k$. Notably, expression 3 is used in Newman's paper [1].

\subsubsection{Expression 4}

Another expression of $g_{0}$ can be found in [9], [25], [30],

$$
\begin{aligned}
g_{0}(r, Z, \omega) & =\frac{2}{\pi} \int_{0}^{\infty}\left(\left(k^{2}-k_{0}^{2}\right) \cos (k Z)+2 k k_{0} \sin (k Z)\right) \frac{K_{0}(k r)}{k^{2}+k_{0}^{2}} d k \\
& -2 \pi k_{0} e^{k_{0} Z} Y_{0}\left(k_{0} r\right)
\end{aligned}
$$

where $K_{0}(k r)$ is the modified Bessel function of the second kind and $Y_{0}\left(k_{0} r\right)$ is the Bessel function of the second kind. This expression is known to be difficult to evaluate numerically due to the singular behavior of $K_{0}(k r)$. It has a logarithmic singular behavior close to $k=0$ and a slowly decaying behavior of the integrand when $r$ is small.

\subsection{Third type}

We define the expressions of the Green function of the third type as:

$$
-4 \pi G_{\infty}(r, Z, \omega)=\frac{1}{R}+\frac{1}{R_{1}}+g_{+}(r, Z, \omega)+2 i \pi k_{0} e^{k_{0} Z} J_{0}\left(k_{0} r\right)
$$

For the expressions of this type, it can be noted that the sum of the contributions of the Rankine and image source term satisfies the homogeneous Neumann boundary condition on the mean free surface $\left(\partial / \partial n(1 / R)+\partial / \partial n\left(1 / R_{1}\right)=0\right)$.

\subsubsection{Expression 5}

Applying Lipschitz's integral into equations (4) or (9), the free-surface term can be written by:

$$
g_{+}(r, Z, \omega)=2 k_{0} P V \int_{0}^{\infty} \frac{1}{k-k_{0}} e^{k Z} J_{0}(k r) d k
$$


Note that it is equivalent to equation (13.15) in Wehausen and Laitone's book[6]. When $r=0$, the free-surface term given can be written:

$$
g_{+}(0, Z, \omega)=-2 k_{0} e^{k_{0} Z} \mathbf{E}_{\mathbf{i}}\left(-k_{0} Z\right)
$$

where $\mathbf{E}_{\mathbf{i}}(x)$ is the exponential integral. When $r=0$ and $Z \rightarrow 0, g_{+}(r, Z, \omega)$ has a logarithmic behavior.

\subsubsection{Expression 6}

In [9], 30], the following expression was given by Havelock:

$$
g_{+}(r, Z, \omega)=-\frac{4 k_{0}}{\pi} \int_{0}^{\infty}\left(k_{0} \cos (k Z)-k \sin (k Z)\right) \frac{K_{0}(k r)}{k^{2}+k_{0}^{2}} d k-2 \pi k_{0} e^{k_{0} Z} Y_{0}\left(k_{0} r\right)
$$

This expression corresponds to equation 3 in Peter and Meylan's paper [25] and to equation $\left(13.17^{\prime \prime \prime}\right)$ in Wehausen's book [6]. One may note the similarity with equation 10 .

\subsubsection{Expression 7}

Expression 7 was proposed by Haskind [31. It corresponds to the equation $\left(13.17^{\prime}\right)$ in Wehausen's Book [6]:

$$
g_{+}(r, Z, \omega)=2 k_{0} e^{k_{0} z_{M}} \int_{\infty}^{z_{M}} \frac{e^{-k_{0} z}}{R_{1}} d z-2 \pi k_{0} e^{k_{0} Z} Y_{0}\left(k_{0} r\right)
$$

\subsubsection{Expression 8}

A modified Haskind Green function was studied by several researchers [8], [9], 10], 32]. It is called the Haskind-Havelock representation in D'elía et al.'s paper [26]. It reads:

$$
g_{+}(r, Z, \omega)=-\pi k_{0} e^{k_{0} Z}\left[\mathbf{H}_{0}\left(k_{0} r\right)+Y_{0}\left(k_{0} r\right)+\frac{2}{\pi} \int_{Z}^{0} \frac{e^{-k_{0} t}}{\sqrt{t^{2}+r^{2}}} d t\right]
$$

where $\mathbf{H}_{0}\left(k_{0} r\right)$ is the Struve function as defined in [33]. This expression is equivalent to equation (3b) in Newman's paper [1] and equation (12) in Liapis's paper [34] where it is called as a Havelock's finite integral. 
It is well suited for evaluating the Green function when $Z$ is small. When the source and field points are both located on the free-surface $(z=0)$, it can be simplified to:

$$
g_{+}(r, 0, \omega)=-\pi k_{0}\left[\mathbf{H}_{0}\left(k_{0} r\right)+Y_{0}\left(k_{0} r\right)\right]+2 i \pi k_{0} J_{0}\left(k_{0} r\right)
$$

215

Substituting (18) into equation 16 leads to:

$$
\begin{aligned}
g_{+}(r, Z, \omega) & =-\pi k_{0} e^{k_{0} Z}\left[\mathbf{H}_{0}\left(k_{0} r\right)+N_{0}\left(k_{0} r\right)+\frac{2}{\pi} \ln \left[k_{0}\left(R_{1}-Z\right)\right]\right. \\
& \left.+\frac{2}{\pi} \int_{Z}^{0} \frac{e^{-k_{0} t}-1}{\sqrt{t^{2}+r^{2}}} d t\right]
\end{aligned}
$$

\subsubsection{Expression 10}

The near-field expression was introduced by Noblesse [14, equation (5.11). It reads:

$$
g_{+}(r, Z, \omega)=-2 \pi k_{0} e^{k_{0} Z} \mathbf{H}_{0}\left(k_{0} r\right)+\frac{4 k_{0}}{\pi} \mathbf{R e}\left[\int_{0}^{\pi / 2} e^{\zeta} \mathbf{E}_{1}(\zeta) d \theta\right]
$$

When the source point and the field point have the same horizontal coordinates (i.e. $r=0$ ), the near-field expression simplifies to:

$$
g_{+}(r=0, Z, \omega)=2 k_{0} e^{k_{0} Z} \mathbf{R e}\left[\mathbf{E}_{1}\left(k_{0} Z+i 0\right)\right]
$$

The near-field expression includes special functions and a finite integral which can be evaluated by direct numerical integration techniques.

\subsubsection{Expression 11}

The far-field expression is given by equation (5.21) in [14. It reads:

$$
g_{+}(r, Z, \omega)=-2 \pi k_{0} e^{k_{0} Z} Y_{0}\left(k_{0} r\right)+\frac{4 k_{0}}{\pi} \mathbf{I m}\left[\int_{0}^{\pi / 2} e^{\zeta} \mathbf{E}_{1}(\zeta) \sec \theta d \theta\right]
$$


Similarly to the near-field expression given in the equation (21), the far-field expression includes special functions and a finite integral which can be evaluated by direct numerical integration techniques.

\subsection{Fourth type}

\subsubsection{Expression 12}

An eigenfunction representation of the Green function was proposed by Peter and Meylan [25]:

$$
\begin{aligned}
-4 \pi G_{\infty}(r, Z, \omega)= & \frac{i k_{0}}{2} e^{k_{0} Z} H_{0}^{(1)}\left(k_{0} r\right)+\frac{1}{\pi^{2}} \int_{0}^{\infty} d k \frac{k^{2}}{k^{2}+k_{0}^{2}} K_{0}(k r) \\
& \times\left\{\cos \left(k z_{M}\right)+\frac{k_{0}}{k} \sin \left(k z_{M}\right)\right\}\left\{\cos \left(k z_{P}\right)+\frac{k_{0}}{k} \sin \left(k z_{P}\right)\right\}
\end{aligned}
$$

$H_{0}^{(1)}$ is the Hankel function of the first kind of first order. There is no singularity in the integrand. Numerical quadrature is used to evaluate the integral in their work.

\subsection{Summary of the analytical expressions}

Expressions 1 to 12 of free-surface Green function are summarized in Table 1. In practice, it is convenient to use non-dimensionalized coordinates rather than dimensional ones since it reduces the integral to a function of only two variables. Therefore, let us define the non-dimensional variables $X=k_{0} r$, $Y=k_{0} Z, Y_{M}=k_{0} z_{M}$ and $Y_{P}=k_{0} z_{P}$. Expressions 1 to 12 can be rewritten as function of these non-dimensional variables given in Table 2 . 


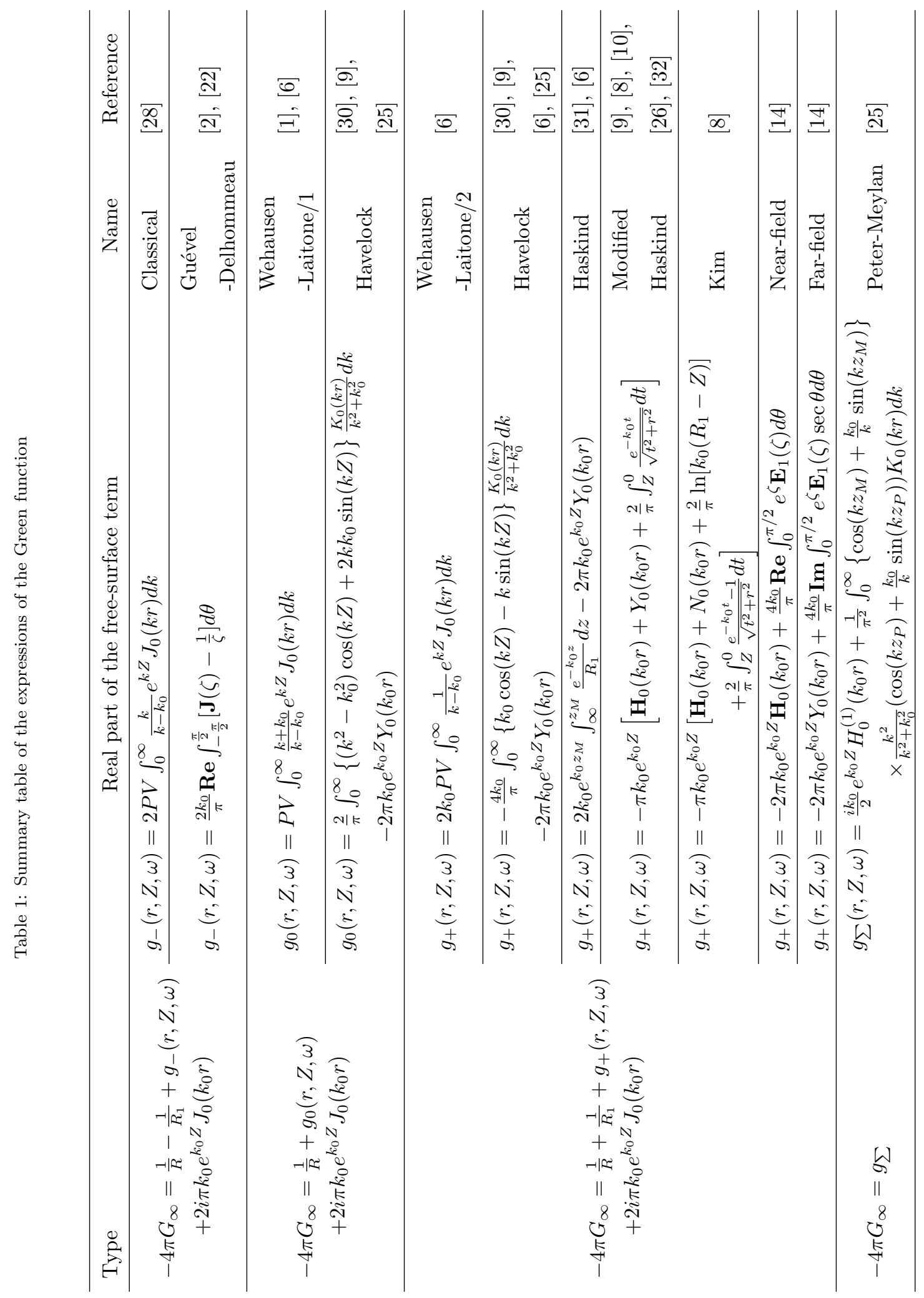




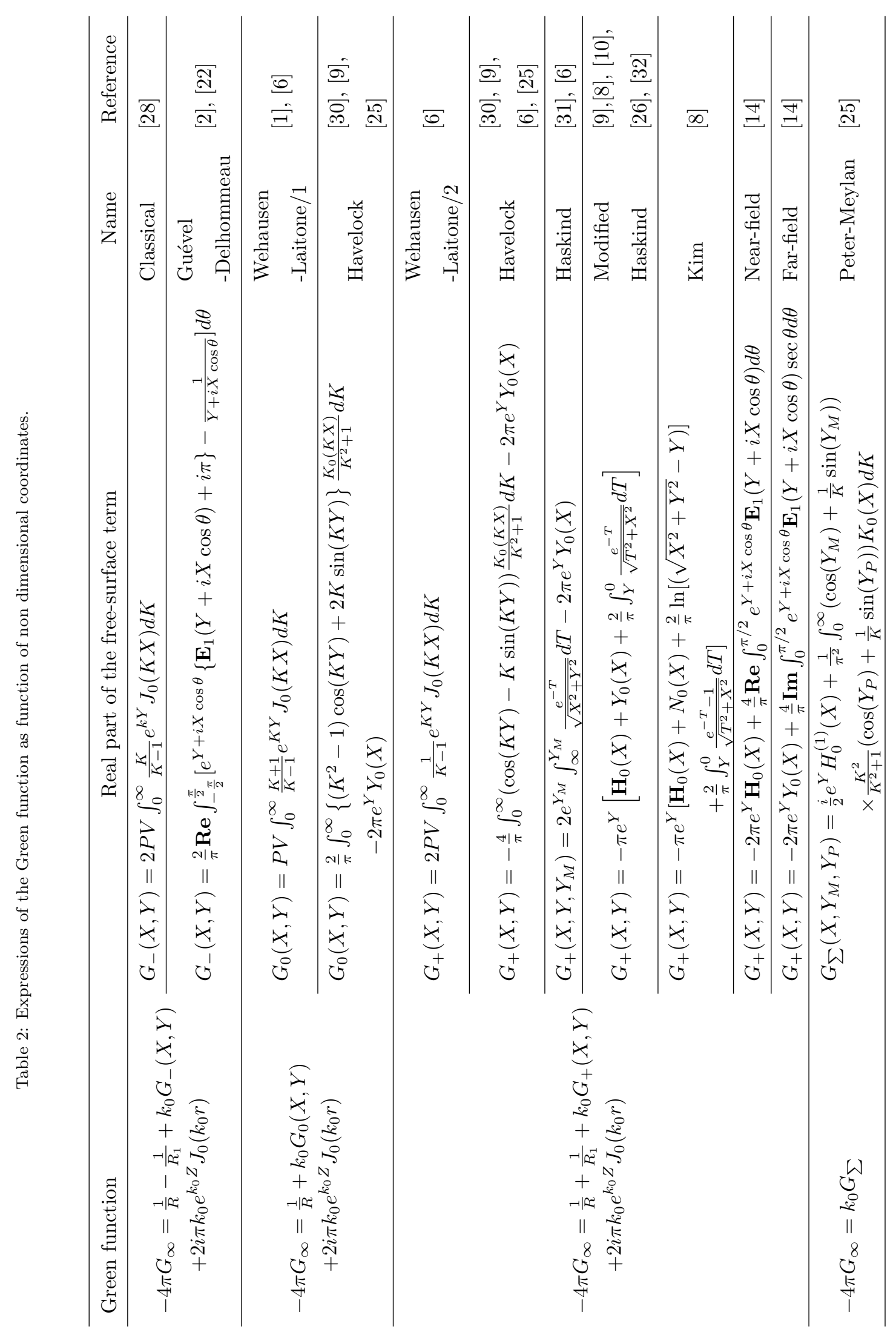




\section{Numerical methods for the evaluation of the Green function and its derivatives}

In this section, the numerical methods for the evaluation of the Green function and its derivatives developed by Newman [1, Delhommeau [2, Telste and Noblesse [3] and Wu et al. [4] are reviewed.

\subsection{Newman's method}

Newman's method proposed in 1985 is based on the non-dimensional versions of $(9)$ and $(16)$. For the efficient evaluation of the integrals in these expressions, Newman divided the quadrant $(X, Y)$ into six sub-domains. Corresponding subcalculated by using the chain rule:

$$
\begin{aligned}
& -4 \pi \frac{\partial G_{\infty}}{\partial r}=\frac{\partial}{\partial r}\left(\frac{1}{R}+\frac{1}{R_{1}}\right)+k_{0} \frac{\partial}{\partial X}\left(k_{0} G_{+}(X, Y)+2 \pi i k_{0} e^{Y} J_{0}(X)\right) \\
& -4 \pi \frac{\partial G_{\infty}}{\partial Z}=\frac{\partial}{\partial Z}\left(\frac{1}{R}+\frac{1}{R_{1}}\right)+k_{0} \frac{\partial}{\partial Y}\left(k_{0} G_{+}(X, Y)+2 \pi i k_{0} e^{Y} J_{0}(X)\right)
\end{aligned}
$$

where $G_{+}(X, Y)$ is the non-dimensionalized free-surface term.

According to equation (16), $\partial G_{+}(X, Y) / \partial Y=-G_{+}(X, Y)-2 / R_{1}$. Therefore, only algorithms for $G_{+}(X, Y)$ and $\partial G_{+}(X, Y) / \partial X$ are required to evaluate the Green function and its derivatives. For the sake of convenience, the finite integral in equation (16) is written as $I(X, Y)$

$$
I(X, Y)=-e^{k_{0} Z} \int_{Z}^{0} \frac{e^{-k_{0} t}}{\sqrt{t^{2}+r^{2}}} d t=\int_{0}^{-Y} \frac{e^{t+Y}}{\sqrt{X^{2}+t^{2}}} d t
$$




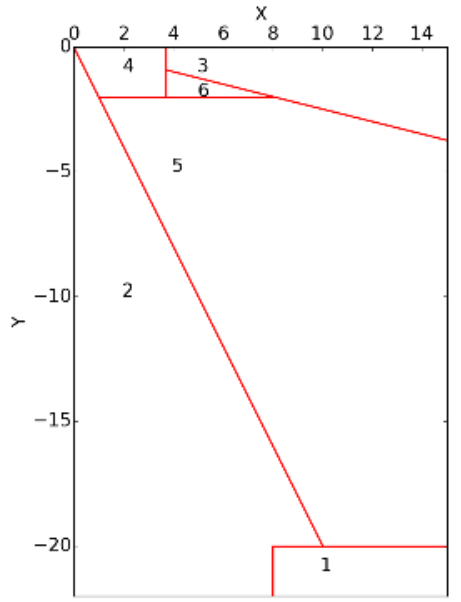

Figure 2: The six sub-domains in Newman's method

\subsubsection{Sub-domain 1}

Sub-domain 1 is defined by $X>8$ and $-Y>20$. There, the non-dimensional version of equation (16) is used. The integral is approximated by Legendre polynomials:

$$
\begin{gathered}
I(X, Y) \approx \sum_{n=0}^{4} n ! P_{n}(\alpha) d^{-(n+1)} \\
\frac{\partial I(X, Y)}{\partial X} \approx \sum_{n=0}^{4}-\frac{n !}{d^{n+3}}\left\{-\frac{X Y}{d} \frac{\partial P_{n}(\alpha)}{\partial \alpha}+(n+1) P_{n}(\alpha) X\right\}
\end{gathered}
$$

where $P_{n}$ is the Legendre polynomial and $(d, \theta)$ denote the polar coordinates such that $X=d \cos \theta$ and $-Y=d \sin \theta$ and $d=k_{0} R_{1}, \alpha=\sin \theta$. According to [1, the truncation order of 4 leads to a 6 decimals accuracy in this sub-domain.

\subsubsection{Sub-domain 2}

For $-X / Y<0.5$, equation $(9)$ is used. The free-surface term $G_{+}(X, Y)$ can be rewritten as:

$$
G_{+}(X, Y)=2 \sum_{n=0}^{\infty} \frac{\left(-X^{2} / 4\right)^{n}}{(n !)^{2}}\left\{\sum_{m=1}^{2 n} \frac{(m-1) !}{(-Y)^{m}}-e^{Y} \mathbf{E}_{\mathbf{i}}(-Y)\right\}
$$




$$
\frac{\partial}{\partial X} G_{+}(X, Y)=2 \sum_{n=0}^{\infty} \frac{2 n}{X} \frac{\left(-X^{2} / 4\right)^{n}}{(n !)^{2}}\left\{\sum_{m=1}^{2 n} \frac{(m-1) !}{(-Y)^{m}}-e^{Y} \mathbf{E}_{\mathbf{i}}(-Y)\right\}
$$

The calculation of $\mathbf{E}_{\mathbf{i}}(-Y)$ depends on the definition in 33. The series are truncated at $n=9$ to achieve a 6 decimals accuracy.

For $X>3.7,-X / Y>4$, the integral is approximated by:

$$
\begin{aligned}
I(X, Y)= & \frac{1}{X} \sum_{n=0}^{\infty}(-1)^{n} \frac{X^{-2 n}}{n !}\left\{\frac{1}{2} \cdot \frac{3}{2} \cdot \frac{5}{2} \ldots \frac{(n-1)}{2}\right\} I_{2 n}(-Y) \\
\frac{\partial I(X, Y)}{\partial X}= & -\frac{1}{X^{2}} \sum_{n=0}^{\infty}(-1)^{n} \frac{X^{-2 n}}{n !}\left\{\frac{1}{2} \cdot \frac{3}{2} \cdot \frac{5}{2} \ldots \frac{(n-1)}{2}\right\} I_{2 n}(-Y) \\
& -\frac{2}{X} \sum_{n=0}^{\infty}(-1)^{n} n \frac{X^{-2 n}}{n ! X}\left\{\frac{1}{2} \cdot \frac{3}{2} \cdot \frac{5}{2} \ldots \frac{(n-1)}{2}\right\} I_{2 n}(-Y)
\end{aligned}
$$

where

$$
I_{0}=1-e^{Y} \quad \text { and } \quad I_{2 n}=\int_{0}^{-Y} e^{t+Y} t^{2 n} d t
$$

The integral $I_{2 n}$ is solution of the recursion relation:

$$
I_{2 n}=(-Y)^{2 n}-2 n(-Y)^{2 n-1}+2 n(2 n-1) I_{2 n-2}
$$

The series in equations (30) and (31) are truncated at $n=3$ to achieve a 6 decimals accuracy.

\subsubsection{Sub-domain 4}

For $0<X<3.7,0<-Y<2, G_{+}(X, Y)$ is approximated using a series expansion [16]:

$$
\begin{aligned}
G_{+}(X, Y) & =-2 e^{Y}\left[J_{0}(X) \log \left\{-Y / X+\left(1+Y^{2} / X^{2}\right)^{1 / 2}\right\}\right. \\
& +\frac{\pi}{2} Y_{0}(X)+\frac{\pi}{2 X} \mathbf{H}_{0}(X)\left(X^{2}+Y^{2}\right)^{1 / 2} \\
& \left.+\left(X^{2}+Y^{2}\right)^{1 / 2} \sum_{m=0}^{\infty} \sum_{n=1}^{\infty} C_{m n} X^{2 m}(-Y)^{n}\right]
\end{aligned}
$$




$$
\begin{aligned}
\frac{\partial}{\partial X} G_{+}(X, Y)=-2 e^{Y}\left[-J_{1}(X) \log \left\{-Y / X+\left(1+Y^{2} / X^{2}\right)^{1 / 2}\right\}\right. \\
+J_{0}(X)\left\{-\frac{Y}{X}+\left(1+\frac{Y^{2}}{X^{2}}\right)^{1 / 2}\right\}^{-1}\left\{\frac{Y}{X^{2}}-\frac{Y^{2}}{X^{3}}\left(1+\frac{Y^{2}}{X^{2}}\right)^{-1 / 2}\right\} \\
\quad-\frac{\pi}{2} Y_{1}(X)-\frac{\pi}{2} \frac{1}{X^{2}} H_{0}(X)\left(X^{2}+Y^{2}\right)^{1 / 2}-\frac{\pi}{2 X} H_{1}(X)\left(X^{2}+Y^{2}\right)^{1 / 2} \\
+\frac{\pi}{2} H_{0}(X)\left(X^{2}+Y^{2}\right)^{-1 / 2}+X\left(X^{2}+Y^{2}\right)^{-1 / 2} \sum_{m=0}^{\infty} \sum_{n=1}^{\infty} C_{m n} X^{2 m}(-Y)^{n} \\
\left.+\left(X^{2}+Y^{2}\right)^{1 / 2} \sum_{m=0}^{\infty} \sum_{n=1}^{\infty} 2 m C_{m n} X^{2 m-1}(-Y)^{n}\right]
\end{aligned}
$$

where the coefficient $C_{m n}$ is defined by:

$$
\begin{aligned}
& C_{0 n}=[(n+1)(n+1) !]^{-1} \\
& C_{m n}=-\left(\frac{n+2}{n+1}\right) C_{m-1, n+2}
\end{aligned}
$$

\subsubsection{Sub-domain 5}

In sub-domain 5 , the finite integral in equation $(16)$ is approximated by:

$$
\begin{gathered}
I(X, Y)=\frac{1}{\sqrt{X^{2}+Y^{2}}}-\frac{e^{Y}}{X}-\frac{Y}{\left(X^{2}+Y^{2}\right)^{3 / 2}} \mathcal{R}(X, Y) \\
\frac{\partial I(X, Y)}{\partial X}=-\frac{X}{\left(X^{2}+Y^{2}\right)^{3 / 2}}+\frac{e^{Y}}{X^{2}}+\frac{3 X Y}{\left(X^{2}+Y^{2}\right)^{5 / 2}} \mathcal{R}(X, Y) \\
-\frac{Y}{\left(X^{2}+Y^{2}\right)^{3 / 2}} \frac{\partial \mathcal{R}(X, Y)}{\partial X}
\end{gathered}
$$

where $\mathcal{R}(X, Y)$ is the slowly-varying residual functions in the given region. $\mathcal{R}(X, Y)$ and $\partial \mathcal{R}(X, Y) / \partial X$ are approximated using Chebyshev polynomials [35. To calculate the coefficients of the Chebyshev polynomials, values of $I$ 
are obtained by direct numerical integration. The Double Chebyshev polynomial expansions are truncated to the desired accuracy by neglecting coefficients smaller than $10^{-6}$.

\subsubsection{Sub-domain 6}

The approximation for the remaining sub-domain is not defined in [1. After numerical tests, the algorithm for sub-domain 3 with $n=7$ is used.

In 1989, Delhommeau introduced an algorithm based on interpolations of the Green function from a pre-calculated table [2]. The Delhommeau's method relies on equation (6). The derivatives are given by:

$$
\begin{aligned}
\frac{\partial G_{\infty}}{\partial r}= & \frac{\partial}{\partial r}\left(\frac{1}{R}+\frac{1}{R_{1}}\right)+\frac{2 k_{0}^{2}}{\pi} \mathbf{R e}\left[\int_{-\pi / 2}^{\pi / 2} i \cos \theta\left[\mathbf{J}(\zeta)-\frac{1}{\zeta}\right] d \theta\right] \\
& +2 i k_{0}^{2} \mathbf{R e}\left[\int_{-\pi / 2}^{\pi / 2} i \cos \theta e^{\zeta} d \theta\right] \\
\frac{\partial G_{\infty}}{\partial Z} & =\frac{\partial}{\partial Z}\left(\frac{1}{R}+\frac{1}{R_{1}}\right)+\frac{2 k_{0}^{2}}{\pi} \mathbf{R e}\left[\int_{-\pi / 2}^{\pi / 2} \mathbf{J}(\zeta) d \theta+\frac{\pi}{k_{0} R_{1}}\right] \\
& +2 i k_{0}^{2} \mathbf{R e}\left[\int_{-\pi / 2}^{\pi / 2} e^{\zeta} d \theta\right]
\end{aligned}
$$

\subsubsection{Elementary functions}

In Delhommeau's method, the expressions of the Green function and its derivatives in equations can be expressed as the function of these four elementary 
integrals:

$$
\begin{aligned}
& D_{1}(X, Y)=\operatorname{Re}\left[\int_{-\pi / 2}^{\pi / 2}(-i \cos \theta)\left[\mathbf{J}(\zeta)-\frac{1}{\zeta}\right] d \theta\right]=\mathbf{I m}\left[\int_{-\pi / 2}^{\pi / 2} \cos \theta\left[\mathbf{J}(\zeta)-\frac{1}{\zeta}\right] d \theta\right] \\
& D_{2}(X, Y)=\operatorname{Re}\left[\int_{-\pi / 2}^{\pi / 2}(-i \cos \theta) e^{\zeta} d \theta\right]=\mathbf{I m}\left[\int_{-\pi / 2}^{\pi / 2} \cos \theta e^{\zeta} d \theta\right] \\
& Z_{1}(X, Y)=\operatorname{Re}\left[\int_{-\pi / 2}^{\pi / 2} \mathbf{J}(\zeta) d \theta\right] \\
& Z_{2}(X, Y)=\operatorname{Re}\left[\int_{-\pi / 2}^{\pi / 2} e^{\zeta} d \theta\right]
\end{aligned}
$$

The algorithm for the calculation of the complex exponential integral function can be found in appendix 3 in [36].

\subsubsection{Tabulation method for near and moderate field}

In Delhommeau's method, the four elementary integrals in equation (41) are interpolated from tabulated data at selected interpolation nodes $\left(X_{i}, Y_{j}\right)$. Lagrange polynomials of the fourth order are used for the interpolation. Direct numerical integration is used for evaluation of the elementary integrals at interpolations nodes. The integration interval $[-\pi / 2, \pi / 2]$ is divided into 5001 points. For the numerical integration, the Simpson's rule is used. The bydefault tabulation domain in AQUADYN 2.1 and NEMOH is $X_{i} \in[0,100]$ and $Y_{j} \in\left[-16,-1.58 \times 10^{-6}\right]$. Since the integrals $D_{1}$ et $Z_{1}$ go to infinity with a logarithmic behavior when $X=0$ and $Y$ tends to $0^{-}$, the interpolation nodes

should be more dense close to zero. Thus, the interpolation nodes $\left(X_{i}, Y_{j}\right)$ are defined by:

$$
\begin{gathered}
X_{i}= \begin{cases}0 & \text { for } \quad i=1 \\
10^{(i-1) / 5-6} & \text { for } \quad i=2,31 \\
4 / 3+(i-32) / 3 & \text { for } \quad i=32,328\end{cases} \\
Y_{j}=\left\{\begin{array}{lll}
-10^{j / 5-6} & \text { for } & j=1,20 \\
-10^{j / 8-4.5} & \text { for } & j=21,46
\end{array}\right.
\end{gathered}
$$


This discretization was found to be sufficient for simple bodies [2]. For complex geometries, refinement of the grid of interpolation nodes may be required.

\subsubsection{Asymptotic formulas for the far field}

For $X>100$ or $-Y>16$, the four elementary integrals are approximated by:

$$
\begin{aligned}
D_{1} & \approx-\frac{\pi X}{k_{0}^{3} R^{3}}+\pi e^{Y} \sqrt{\frac{2 \pi}{X}}\left\{\cos \left(X-\frac{\pi}{4}\right)-\frac{1}{2 X} \sin \left(X-\frac{\pi}{4}\right)\right\} \\
D_{2} & \approx e^{Y} \sqrt{\frac{2 \pi}{X}}\left\{\sin \left(X-\frac{\pi}{4}\right)+\frac{1}{2 X} \cos \left(X-\frac{\pi}{4}\right)\right\} \\
Z_{1} & \approx \frac{\pi Y}{k_{0}^{3} R^{3}}-\pi e^{Y} \sqrt{\frac{2 \pi}{X}} \sin \left(X-\frac{\pi}{4}\right)-\frac{\pi}{k_{0} R_{1}} \\
Z_{2} & \approx e^{Y} \sqrt{\frac{2 \pi}{X}} \cos \left(X-\frac{\pi}{4}\right)
\end{aligned}
$$

\subsection{Telste-Noblesse's method}

In the algorithm of Telste-Noblesse, three representations are used: The modified Haskind representation (equation (16)), the near-field representation (equation (21) and the far-field representation (equation (22)). The derivatives of Green function are expressed as the following:

$$
\begin{aligned}
& \frac{\partial G_{\infty}(r, Z, \omega)}{\partial r}=\frac{\partial}{\partial r}\left(\frac{1}{R}+\frac{1}{R_{1}}\right)-2 k_{0}^{2} \frac{\partial G_{+}(X, Y)}{\partial X}-\pi i k_{0}^{2} e^{Y} J_{1}(X) \\
& \frac{\partial G_{\infty}(r, Z, \omega)}{\partial Z}=\frac{\partial}{\partial Z}\left(\frac{1}{R}+\frac{1}{R_{1}}\right)+2 k_{0}^{2}\left\{\frac{1}{k_{0} R_{1}}+G_{+}(X, Y)\right\}+\pi i k_{0}^{2} e^{Y} J_{0}(X)
\end{aligned}
$$

Depending on the representation, the derivatives with respect to $X$ of real part of the free-surface term can be written:

$$
\begin{aligned}
& \frac{\partial G_{+}(X, Y)}{\partial X}=-\pi\left[\frac{2}{\pi}-\mathbf{H}_{1}(X)-Y_{1}(X)\right] e^{Y}+2 X \int_{0}^{-Y} e^{(t+Y)} \frac{1}{\sqrt{t^{2}+X^{2}}} d t \\
& \frac{\partial G_{+}(X, Y)}{\partial X}=-2 \pi\left[\frac{2}{\pi}-\mathbf{H}_{1}(X)\right] e^{Y}-\frac{4}{\pi} \mathbf{I m}\left[\int_{0}^{\pi / 2}\left[e^{\zeta} \mathbf{E}_{1}(\zeta)-\frac{1}{\zeta}\right] \cos \theta d \theta\right] \\
& \frac{\partial G_{+}(X, Y)}{\partial X}=2 \pi Y_{1}(X) e^{Y}+\frac{4}{\pi} \mathbf{R e}\left[\int_{0}^{\pi / 2}\left[e^{\zeta} \mathbf{E}_{1}(\zeta)-\frac{1}{\zeta}\right] \sec ^{2} \theta d \theta\right]
\end{aligned}
$$


In the algorithm of Telste-Noblesse, the computational domain is also split into sub-domains. The sub-domains are shown in Fig, 3 .

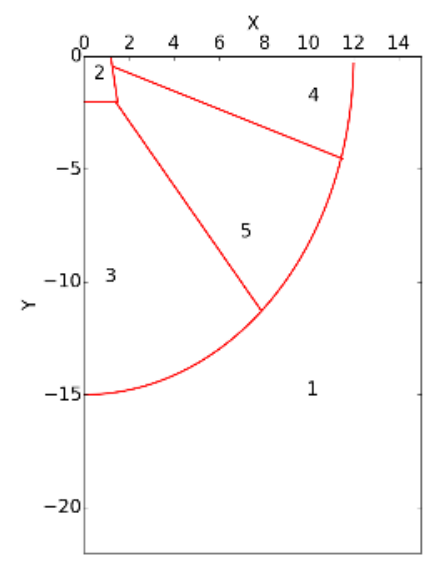

Figure 3: Sub-domains used in the Telste-Noblesse's method

\subsubsection{Sub-domain 1}

For large to moderate values of $d$ and $X \neq 0$, an asymptotic expansion is applied for the far-field representation. For $0 \geq Y \geq Y_{t}\left(Y_{t}\right.$ being a user-defined variable set to -14.5 in the Telste-Noblesse's algorithm), the free-surface term 340 is expanded as follows:

$$
G_{+}(X, Y) \approx-2 \pi \mathbf{Y}_{0}(X) e^{Y}-2 \sum_{n=0}^{\infty} \frac{p_{n}(\alpha)}{d^{n+1}}
$$

$\alpha=-Y / d(0 \leq \alpha<1)$ and the polynomials $p_{n}$ are defined by:

$$
\begin{aligned}
& p_{0}(\alpha)=1 \\
& p_{1}(\alpha)=\alpha \\
& p_{n}(\alpha)=(2 n-1) \alpha p_{n-1}(\alpha)-(n-1)^{2} p_{n-2}(\alpha) \text { for } n \geq 2
\end{aligned}
$$

The derivatives with respect to $X$ are given by:

$$
\frac{\partial G_{+}(X, Y)}{\partial X} \approx 2 \pi \mathbf{Y}_{1}(X) e^{Y}+2 X \sum_{n=0}^{\infty} \frac{q_{n}(\alpha)}{d^{n+3}}
$$


where the polynomials $q_{n}$ are defined by:

$$
\begin{aligned}
& q_{0}(\alpha)=1 \\
& q_{1}(\alpha)=3 \alpha \\
& q_{n}(\alpha)=(2 n+1) \alpha q_{n-1}(\alpha)-(n+1)(n-1) q_{n-2}(\alpha) \text { for } n \geq 2
\end{aligned}
$$

When $Y_{t} \geq Y$, the wave term and its derivatives are approximated as follows:

345

$$
\begin{gathered}
G_{+}(X, Y) \approx-2 \sum_{n=0}^{\infty} \frac{p_{n}(\alpha)}{d^{n+1}} \quad \text { for } \quad Y \rightarrow-\infty \\
\frac{\partial G_{+}(X, Y)}{\partial X} \approx 2 X \sum_{n=0}^{\infty} \frac{q_{n}(\alpha)}{d^{n+3}} \quad \text { for } \quad Y \rightarrow-\infty
\end{gathered}
$$

The truncation order of the series is $n=10$ outside the ellipse $X^{2} / 12^{2}+$ $Y^{2} / 15^{2}=1$ and $n=5$ outside the ellipse $X^{2} / 16^{2}+Y^{2} / 20^{2}=1$. The absolute errors are reported to be smaller than $10^{-6}$ in $[3]$.

\subsubsection{Sub-domain 2}

The sub-domain 2 corresponds to $0 \leq X \leq 1.2-0.15 Y,-2 \leq Y \leq 0$. A convergent ascending series is used. The ascending series is based on the near-field representation. The free-surface term is expressed as:

$$
G_{+}(X, Y)=2\left[\left\{-\ln (d-Y)+F_{0}(Y)\right\} J_{0}(X)+2 S_{0}(X, Y)\right] e^{Y}+2 T_{0}(X, Y)
$$

where $F_{0}(Y), S_{0}(X, Y), T_{0}(X, Y)$ are defined as follows:

$$
\begin{aligned}
F_{0}(Y) & =\ln 2-\gamma-\sum_{n=1}^{\infty} \frac{(-Y)^{2}}{n \cdot n !} \\
S_{0}(X, Y) & =\sum_{n=1}^{\infty}\left\{\sum_{k=0}^{n^{\prime}}(-1)^{k} \frac{\sigma^{n-2 k}}{(n-2 k) k !(n-k) !}\right\}\left(-\frac{X}{2}\right)^{n} \\
T_{0}(X, Y) & =\sum_{n=1}^{\infty} F_{n}(Y)\left(-\frac{X^{2}}{4}\right)^{n}
\end{aligned}
$$

In these last equations, $\gamma=0.5772 \ldots$ is the Euler's constant. $n^{\prime}$ is the largest integer which does not exceed $(n-1) / 2$ and $\sigma=X /(d-Y) . F_{n}(Y)$ is defined 
as follows:

$$
F_{n}(Y)=\frac{1}{n ! n !} \sum_{k=0}^{\infty}\left\{\sum_{m=1}^{2 n} \frac{1}{m+k}\right\} \frac{Y^{k}}{k !}
$$

A corresponding ascending series for the derivatives with respect to $X$ is given by:

$$
\begin{aligned}
\frac{\partial G_{+}(X, Y)}{\partial X}= & -2 X \frac{J_{0}(X)}{d(d-Y)}-2\left\{-\ln (d-Y)+F_{0}(Y)\right\} J_{1}(X) \\
& -2 S_{1}(X, Y) e^{Y}-2 T_{1}(X, Y)
\end{aligned}
$$

360

with

$$
\begin{aligned}
& S_{1}(X, Y)=\frac{X}{d}+\sum_{n=2}^{\infty}\left[\sum_{k=0}^{n^{\prime}}(-1)^{k} \sigma^{n-2 k} \frac{n /(n-2 k)-Y / d}{k !(n-k) !}\right]\left(-\frac{X}{2}\right)^{n-1} \\
& T_{1}(X, Y)=\frac{X}{2} \sum_{n=1}^{\infty} n F_{n}(Y)\left(-\frac{X^{2}}{4}\right)^{n-1}
\end{aligned}
$$

5 terms are required for the series $T_{0}$ and $T_{1}$ and 10 terms are required for the series $S_{0}$ and $S_{1}$ to achieve $10^{-6}$ accuracy. For $X<0.55-2 Y$, less terms are needed.

\subsubsection{Sub-domain 3}

365 A Taylor series expansion of the near-field integral representation is used in the neighborhood of the vertical axis $X=0$ :

$$
G_{+}(X, Y)=2 J_{0}(X)\left\{e^{Y} \boldsymbol{R e}\left[\mathbf{E}_{1}(Y+i 0)\right]\right\}+2 \sum_{n=1}^{\infty} r_{n}(Y)\left(-\frac{X^{2}}{4 Y^{2}}\right)^{n}
$$

$r_{n}(Y)$ is given by:

$$
r_{n}(Y)=\left[1 /(n !)^{2}\right] \sum_{k=0}^{2 n-1}(2 n-1-k) !(-Y)^{k}
$$

It is also solution of the recursion relation:

$$
r_{n}(Y)=(2 n-2) !(2 n-1-Y)(n !)^{2}+\left(Y^{2} / n^{2}\right) r_{n-1}(Y), n \geq 2
$$


Similarly, a Taylor series expansion can be obtained for the derivatives:

$\frac{\partial G_{+}(X, Y)}{\partial X}=-2 J_{1}(X)\left\{e^{Y} \mathbf{R e}\left[\mathbf{E}_{1}(Y+i 0)\right]\right\}-\frac{X}{Y^{2}} \sum_{n=1}^{\infty} n \cdot r_{n}(Y)\left(-\frac{X^{2}}{4 Y^{2}}\right)^{n-1}$

370 as in 3. To achieve the accuracy of $10^{-6}$, five, ten and fifteen terms are respectively retained in the series depending on whether $X \leq \min (-0.3 Y, 1.8)$, $X \leq \min (-0.5 Y, 5.4)$ or $X \leq \min (-0.7 Y, 9.1)$ with $2 \leq-Y \leq 15$.

\subsubsection{Sub-domain 4}

375 of the Haskind integral representation is used :

$$
G_{+}(X, Y)=\pi\left\{-\mathbf{H}_{0}(X)-Y_{0}(X)\right\} e^{Y}+2 \sum_{n=1}^{\infty} s_{n}(X)\left(\frac{Y}{X}\right)^{n}
$$

The polynomials $s_{n}$ are solution of the following recursion relation:

$$
\begin{aligned}
s_{1}(X) & =1 \\
s_{2 n}(X) & =\frac{X}{2 n} s_{2 n-1}(X), \quad \text { for } n \geq 1 \\
s_{2 n+1}(X) & =\left\{X s_{2 n}(X)+C_{n}\right\} /(2 n+1), \quad \text { for } n \geq 1
\end{aligned}
$$

with

$$
C_{n}=(-1)^{n}\{1 \cdot 3 \cdot 5 \cdots(2 n-1)\} /\{2 \cdot 4 \cdot 6 \cdots(2 n)\}
$$

For the derivative, the Taylor series expansion is:

$$
\frac{\partial G_{+}(X, Y)}{\partial X}=-\pi\left\{\frac{2}{\pi}-\mathbf{H}_{1}(X)-Y_{1}(X)\right\} e^{Y}-\frac{2}{X} \sum_{n=1}^{\infty} t_{n}(X)\left(\frac{Y}{X}\right)^{n}
$$

380

with

$$
\begin{aligned}
t_{1}(X) & =1 \\
t_{2 n}(X) & =X t_{2 n-1}(X) / 2 n, \quad \text { for } n \geq 1 \\
t_{2 n+1}(X) & =X t_{2 n}(X) /(2 n+1)+C_{n}, \quad \text { for } n \geq 1
\end{aligned}
$$

In the region $1.2 \leq X \leq 12$, the truncation order is 10 if $Y \geq \max (-0.4 X+$ $0.15,-1.5)$ or 20 if $Y \geq-0.4 X$. 


\subsubsection{Sub-domain 5}

The sub-domain 5 corresponds to the region where $1<d<10$ along with is used. The free-surface term and its derivatives are given by:

$$
\begin{gathered}
G_{+}(X, Y)=\pi\left\{-\mathbf{H}_{0}(X)-Y_{0}(X)\right\} e^{Y}-2\left[F_{0}(X)+I_{0}(X)\right] e^{Y+c X} \\
\frac{\partial G_{+}(X, Y)}{\partial X}=-\pi\left\{\frac{2}{\pi}-\mathbf{H}_{1}(X)+Y_{1}(X)\right\} e^{Y}-2\left[F_{1}(X)+I_{1}(X)\right] e^{Y+c X} / X
\end{gathered}
$$

where $c$ is a special constant and $F_{0}(X), F_{1}(X), I_{0}(X, Y)$ and $I_{1}(X, Y)$ are defined by the integrals:

$$
F_{0}(X)=\int_{0}^{c} e^{-X(c-\tau)}\left(1+\tau^{2}\right)^{-1 / 2} d \tau
$$

390

$$
\begin{gathered}
F_{1}(X)=\int_{0}^{c} e^{-X(c-\tau)}\left(1+\tau^{2}\right)^{-3 / 2} d \tau \\
I_{0}(X, Y)=\int_{c}^{-Y / X} e^{-X(c-\tau)}\left(1+\tau^{2}\right)^{-1 / 2} d \tau \\
I_{1}(X, Y)=\int_{c}^{-Y / X} e^{-X(c-\tau)}\left(1+\tau^{2}\right)^{-3 / 2} d \tau
\end{gathered}
$$

These integrals involve the integrands $\left(1+\tau^{2}\right)^{-1 / 2}$ and $\left(1+\tau^{2}\right)^{-3 / 2}$. Those terms can be approximated by polynomials. Then the integrals can be integrated analytically which leads to series expansion forms of the solution. The special constant $c$ is set to $0.4,0.65,0.85$ and 1.1 for the regions $0.4<-Y / X \leq$ $0.65,0.65<-Y / X \leq 0.85,0.85<-Y / X \leq 1.1$ and $1.1<-Y / X \leq 10 / 7$, respectively.

\subsection{Wu et al.'s method}

400

A simple approximation of the Green function was proposed by $\mathrm{Wu}$ et al. [4] which valid in the whole domain. It derives from the Noblesse's near-field 
representation [13. The free-surface term is split into a local flow term $L(X, Y)$ and an out-going free-surface term $W(X, Y)$ :

$$
G_{0}(X, Y)=-\{L(X, Y)+W(X, Y)\}
$$

with

$$
\begin{aligned}
& L(X, Y)=-\frac{1}{k_{0} R_{1}}-\frac{4}{\pi} \mathbf{R e}\left[\int_{0}^{\pi / 2} e^{\zeta} \mathbf{E}_{1}(\zeta) d \theta\right] \\
& W(X, Y)=2 \pi \mathbf{H}_{0}(X) e^{Y}
\end{aligned}
$$
function are:

$$
\begin{aligned}
\frac{\partial G_{\infty}}{\partial r} & =\frac{\partial}{\partial r}\left(\frac{1}{R}\right)-k_{0}\left(\frac{\partial L}{\partial X}+\frac{\partial W}{\partial X}\right) \\
\frac{\partial G_{\infty}}{\partial Z} & =\frac{\partial}{\partial Z}\left(\frac{1}{R}\right)-k_{0}\left(\frac{\partial L}{\partial Y}+W\right)
\end{aligned}
$$

with

$$
\begin{aligned}
& \frac{\partial L}{\partial Y}(X, Y)=\frac{Y}{k_{0}^{3} R_{1}^{3}}-\frac{1}{k_{0} R_{1}}+L(X, Y) \\
& \frac{\partial W}{\partial X}(X, Y)=2 \pi\left[2 / \pi-\mathbf{H}_{1}(X)\right] e^{Y} \\
& \frac{\partial L}{\partial X}(X, Y)=\frac{X}{k_{0}^{3} R_{1}^{3}}+L_{*}(X, Y)
\end{aligned}
$$

and

$$
L_{*}(X, Y)=\frac{4}{\pi} \mathbf{I m}\left[\int_{0}^{\frac{\pi}{2}}\left[e^{\zeta} \mathbf{E}_{1}(\zeta)-\frac{1}{\zeta}\right] \cos \theta d \theta\right]
$$

Wu et al. developed a simple approximation for the local flow term and its derivative: $L \approx L^{a}, L_{*} \approx L_{*}^{a}$. The approximated local flow term $L^{a}$ is:

$$
L^{a}=-\frac{1}{k_{0} R_{1}}+\frac{2 L_{P}}{1+k_{0}^{3} R_{1}^{3}}+2 \rho(1-\rho)^{3} L_{R}
$$

with

$$
\begin{aligned}
& L_{P}=e^{Y}\left(\log \frac{k_{0} R_{1}-Y}{2}+\gamma-2 k_{0}^{2} R_{1}^{2}\right)+k_{0}^{2} R_{1}^{2}-Y \\
& L_{R}=(1-\beta) A-\beta B-\frac{\alpha C}{1+6 \alpha \rho(1-\rho)}+\beta(1-\beta) D
\end{aligned}
$$


where $\alpha=-Z / R_{1}, \beta=r / R_{1}, \rho=k_{0} R_{1} /\left(1+k_{0} R_{1}\right)$ and the polynomials $A, B, C$ and $D$ are defined by:

$$
\begin{aligned}
A & =1.21-13.328 \rho+215.896 \rho^{2}-1763.96 \rho^{3}+8418.94 \rho^{4}-24314.21 \rho^{5} \\
& +42002.57 \rho^{6}-41592.9 \rho^{7}+21859 \rho^{8}-4838.6 \rho^{9} \\
B & =0.938+5.373 \rho-67.92 \rho^{2}+796.534 \rho^{3}-4780.77 \rho^{4}+17137.74 \rho^{5} \\
& -36618.81 \rho^{6}+44894.06 \rho^{7}-29030.24 \rho^{8}+7671.22 \rho^{9} \\
C & =1.268-9.747 \rho+209.653 \rho^{2}-1397.89 \rho^{3}+5155.67 \rho^{4}-9844.35 \rho^{5} \\
& +9136.4 \rho^{6}-3272.62 \rho^{7} \\
D & =0.632-40.97 \rho+667.16 \rho^{2}-6072.07 \rho^{3}+31127.39 \rho^{4}-96293.05 \rho^{5} \\
& +181856.75 \rho^{6}-205690.43 \rho^{7}+128170.2 \rho^{8}-33744.6 \rho^{9}
\end{aligned}
$$

The other term $L_{*}^{a}$ is:

$$
L_{*}^{a}=\frac{2 L_{P *}}{1+k_{0}^{3} R_{1}^{3}}-4 L_{Q *}+2 \rho(1-\rho)^{3} L_{R *}
$$

415 with

$$
\begin{aligned}
& L_{P *}=\frac{\beta+X}{k_{0} R_{1}-Y}-2 \beta+2 e^{Y} k_{0} R_{1}-X \\
& L_{Q *}=e^{-k_{0} R_{1}}(1-\beta)\left(1+\frac{k_{0} R_{1}}{1+k_{0}^{3} R_{1}^{3}}\right) \\
& L_{R *}=\beta A_{*}-(1-\alpha) B_{*}+\beta(1-\beta) \rho(1-2 \rho) C_{*}
\end{aligned}
$$

The polynomials $A_{*}, B_{*}$ and $C_{*}$ are defined by:

$$
\begin{aligned}
A_{*} & =2.948-24.53 \rho+249.69 \rho^{2}-754.85 \rho^{3}-1187.71 \rho^{4}+16370.75 \rho^{5} \\
& -48811.41 \rho^{6}+68220.87 \rho^{7}-46688 \rho^{8}+12622.25 \rho^{9} \\
B_{*}= & 1.11+2.894 \rho-76.765 \rho^{2}+1565.35 \rho^{3}-11336.19 \rho^{4}+44270.15 \rho^{5} \\
& -97014.11 \rho^{6}+118879.26 \rho^{7}-76209.82 \rho^{8}+19923.28 \rho^{9} \\
C_{*} & =14.19-148.24 \rho+847.8 \rho^{2}-2318.58 \rho^{3}+3168.35 \rho^{4}-1590.27 \rho^{5}
\end{aligned}
$$

\subsection{Summary of numerical schemes}

The mathematical formulas for the calculation of the free-surface term of the Green function and its derivative for the four methods are summarized in

Table 3 and Table 4 


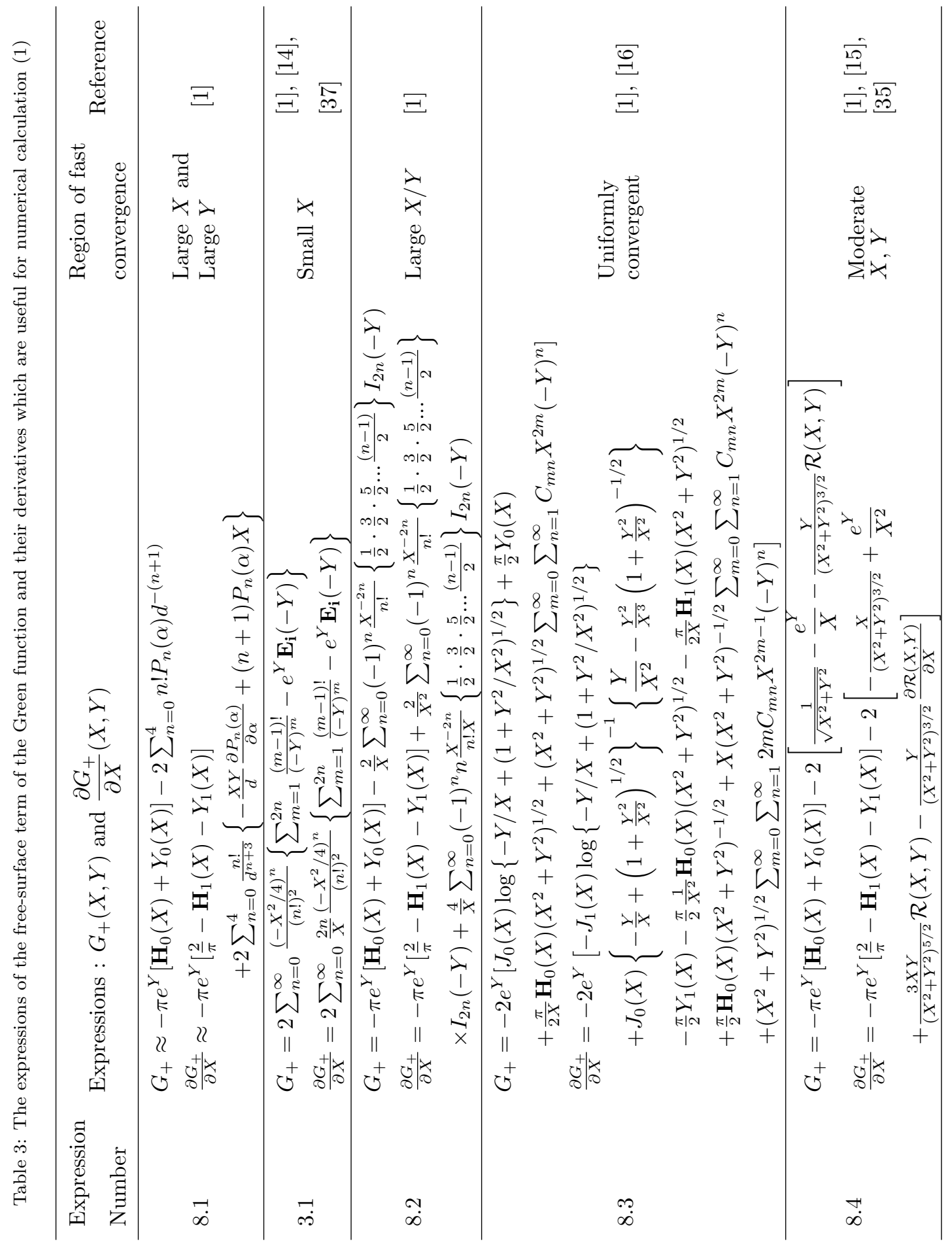




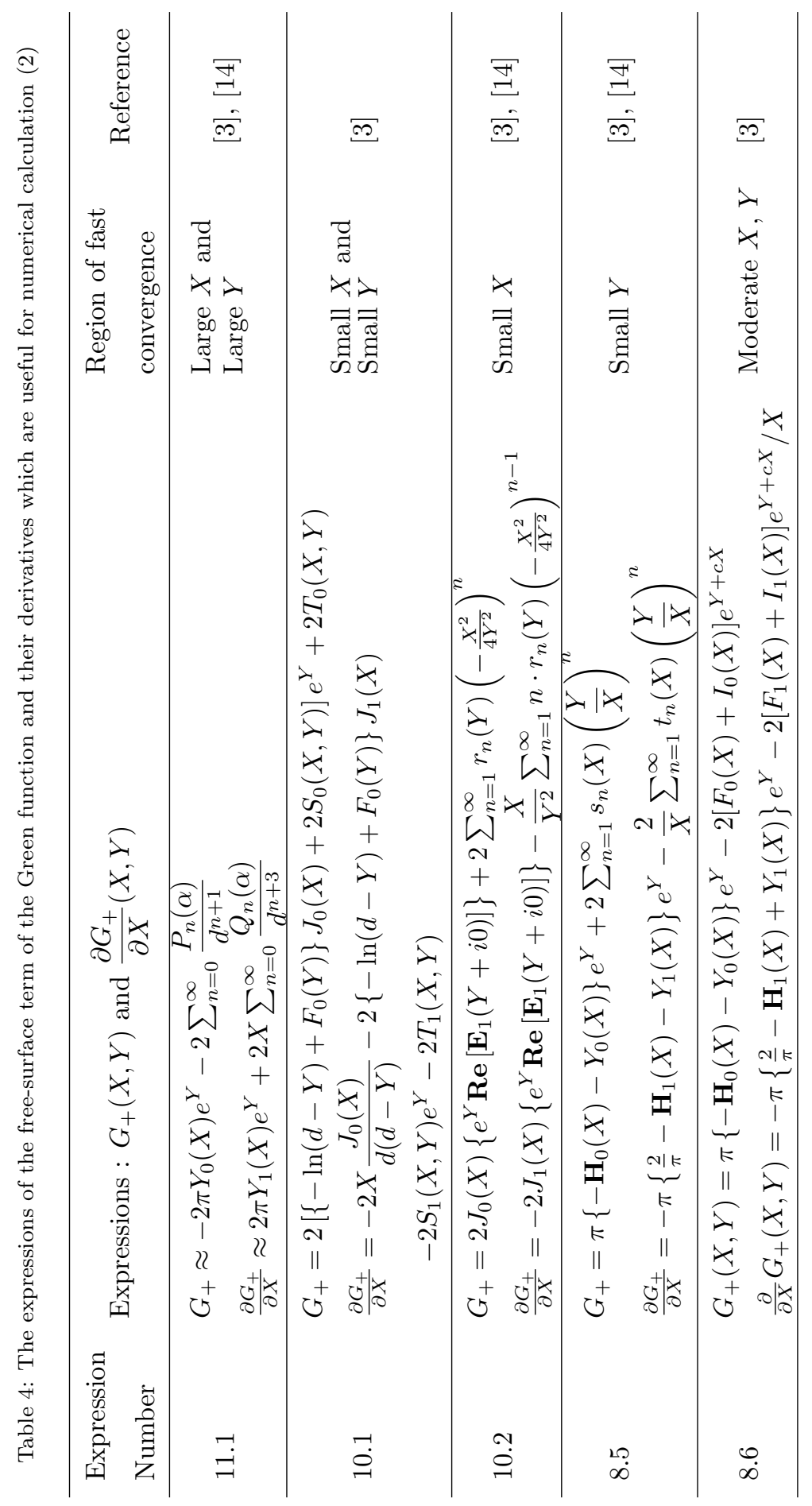




\section{Comparison of the four methods}

We implemented the algorithms of Newman, Delhommeau, Telste and Noblesse and Wu et al. For the Telste-Noblesse's method, the source code is given in their paper [3. However, it should be noted that we changed the precision lows the NEMOH code (which is open source) with a few minor modifications in the tabulated file. For the Newman's and Wu et al.'s methods, we developed the source code based on their papers. It should be noted that the computational time may vary depending on the coding technique.

430 6700 CPU @ 3.40 GHZ. The codes were written in FORTRAN90 and compiled with intel fortran 14.0.1. The evaluation of the Struve function is based on the algorithm of Newman 38. For the Bessel functions, the functions available in the FORTRAN compiler were used.

$X \in[0.001,22], Y \in[0.001,15]$. This domain is expected to cover most of the practical cases. The case of the source and field points located at the same position is not considered since it corresponds to the singularities. Analytical expressions are available for $Y=0$ (equation (16))and for $X=0$ (equation 445 (13)).

Reference values for the real part of the non-dimensionalized free-surface term of the Green function $\left(G_{-}\right)$and its derivative $\left(G_{-X}=\partial G_{-} / \partial X\right)$ are shown in Fig. 4. They were obtained by direct numerical integration using an adaptive Gaussian 3-point quadrature. The absolute error for the reference values is less than $10^{-8}$. 


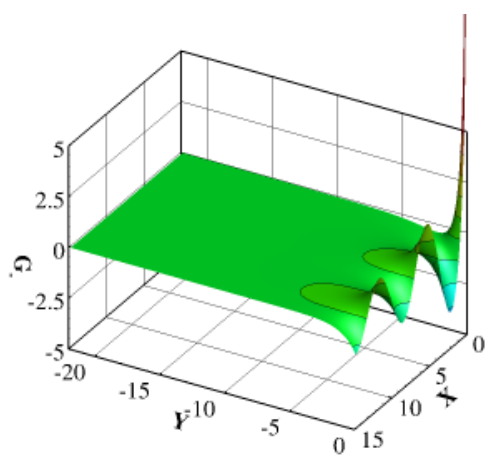

(a) G

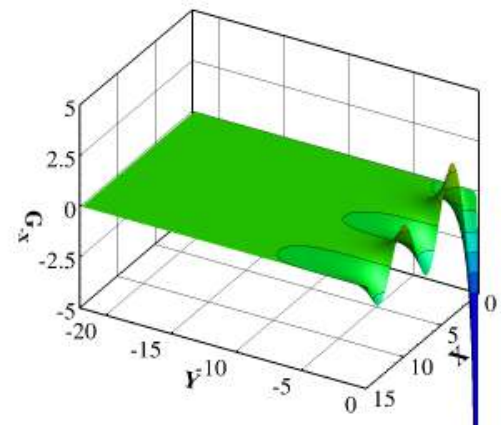

(b) $G_{-X}$

Figure 4: The free-surface term of the Green function $G_{-}$and its derivative $G_{-X}$ using the direct integral method

The absolute error for the Green function between the values computed from the direct integration and four methods are plotted in Fig. 5. As expected, a 6 decimals accuracy is obtained with the Newman's method over the whole computational domain, see Fig 5 (a) . The error is greater in the sub-domain 1, domain except when $(X, Y)$ is close to $(0,0)$. However, the value of the Green function is large in that situation which makes the relative error small, in the order of 1 to $2 \%$.

Note that for some cases of $(X, Y)$, the accuracy of the derivative can be 


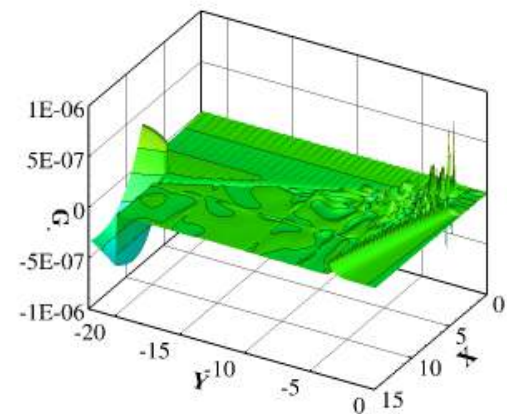

(a) Newman's method

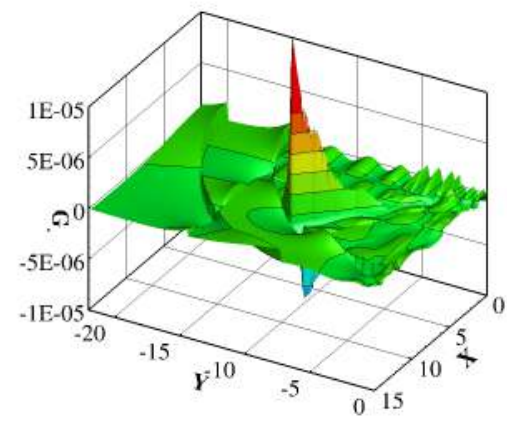

(c) Telste and Noblesse's method

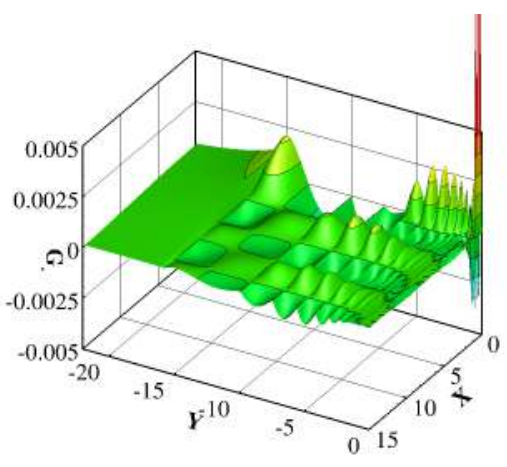

(b) Delhommeau's method

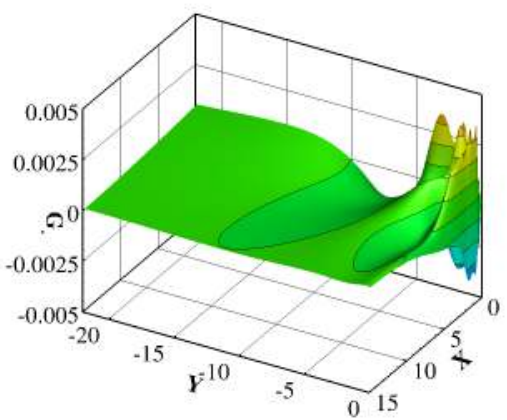

(d) Wu et al.'s method

Figure 5: The errors of the free-surface term of the Green function $G_{-}$of Newman's, Delhommeau's, Telste and Noblesse's, Wu et al.'s methods compared to direct integral method depending on the method.

The computational time for calculating the Green functions and its derivative over the set of discretized $(X, Y)$ values for the different algorithms is shown in Table 5. As one can expect, the computational time is the greatest for the methods that are the most accurate (direct numerical integration and Newman's method). The direct numerical integration with tolerance of $10^{-8}$ needs 20 times more computational time than the Newman's method. The Delhommeau and Telste-Noblesse's methods are an order of magnitude faster than the Newman's method. However, the accuracy is respectively about three orders of magnitude 


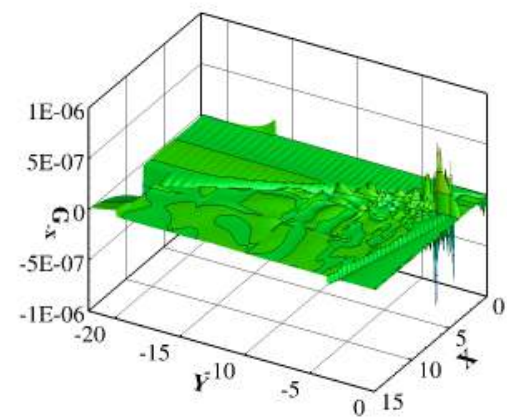

(a) Newman's method

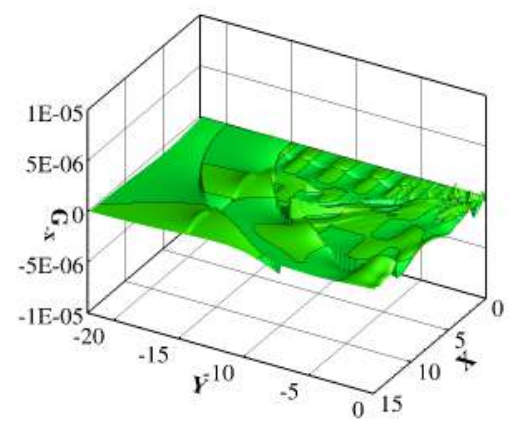

(c) Telste and Noblesse's method

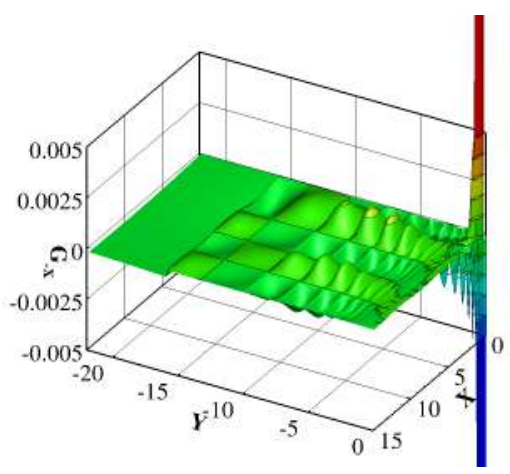

(b) Delhommeau's method

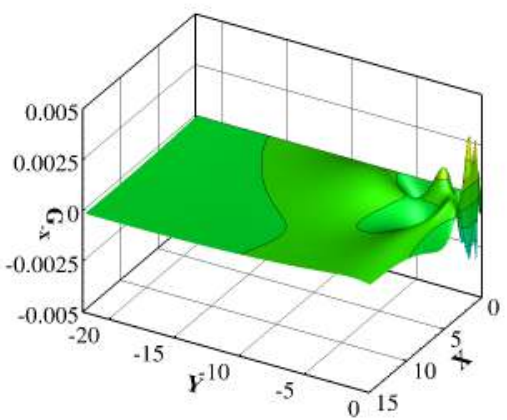

(d) Wu et al.'s method

Figure 6: The errors of the derivative of free-surface term of the Green function $G_{-X}$ of Newman's, Delhommeau's, Telste and Noblesse's, Wu et al. methods compared to direct integral method

and slightly less than the Newman's method. With respect to computational time, the Wu et al.'s method is the fastest one.

Overall, it appears that the best method is the Newman's method and TelsteNoblesse's method with respect to accuracy. With respect to computational time, the Wu et al.'s method is best.

As in Newman's and Telste-Noblesse's method, the evaluations algorithms are different in each sub-domains. The computational time for each $(X, Y)$ computational points as function of the method is shown in Fig. 7. It can be seen that the computational time depends not only on the method but also 
Table 5: Computational time for calculating the Green function and its derivatives a function of the different algorithms

\begin{tabular}{|c|c|c|}
\hline Algorithm & $\begin{array}{c}\text { Total computation time }(\mathrm{s}) \\
\left(N_{X}=220, N_{Y}=150\right)\end{array}$ & $\begin{array}{c}\text { Averaged } \\
\text { computation time (s) }\end{array}$ \\
\hline Direct numerical integration & $3.840 \times 10^{-1}$ & $1.164 \times 10^{-5}$ \\
\hline Newman & $1.896 \times 10^{-2}$ & $5.745 \times 10^{-7}$ \\
\hline Delhommeau & $1.908 \times 10^{-3}$ & $5.782 \times 10^{-8}$ \\
\hline Telste-Noblesse & $1.532 \times 10^{-3}$ & $4.642 \times 10^{-8}$ \\
\hline Wu et al. & $4.920 \times 10^{-5}$ & $1.491 \times 10^{-9}$ \\
\hline
\end{tabular}

on the sub-domains. The local computational time for the Newman's method is shown in Fig 7(a), The average computational time for each sub-domain is shown in Table 6. In the sub-domain 2, the computational time increases with increasing $Y$.

The computational time for the Delhommeau's method is shown in Fig 7(b) Since it is based on the fourth order Lagrange polynomial interpolation, the computational time does not vary much over the interpolation domain. For large $X$ and $Y$, the asymptotic formulation is used to calculate the Green function which explains why the computational time for $Y \geq 16$ is much shorter than for the rest of the domain.

The compuational time for the Telste-Noblesse's method in shown in Fig. $5007(\mathrm{c})$. The computational time varies between $O\left(10^{-7}\right)$ and $O\left(10^{-8}\right)$. The average computational time for each sub-domain is shown in table 7. For any given $X$ and $Y$, the computational time appears to be less than that of the Newman's method.

The computational time for the Wu et al.'s method is shown in Fig. 7(d) 505 It is shown that the computational time suddenly increases for $X \geq 3$. It is because despite the fact that the flow term is approximated with a simple polynomials with the elementary functions, the Wu et al.'s method still requires a 
subroutine to calculate the Struve function $\left(\mathbf{H}_{0}\left(k_{0} r\right)\right)$. This subroutine is based on the algorithm by Newman [39], which uses different approaches depending on whether $X$ is greater or smaller than 3. Nevertheless, a key feature of the $\mathrm{Wu}$ et al.'s method is that it is not based on subdivisions of the flow domain. Thus, it may be more suited for parallel computations compared to the other methods.

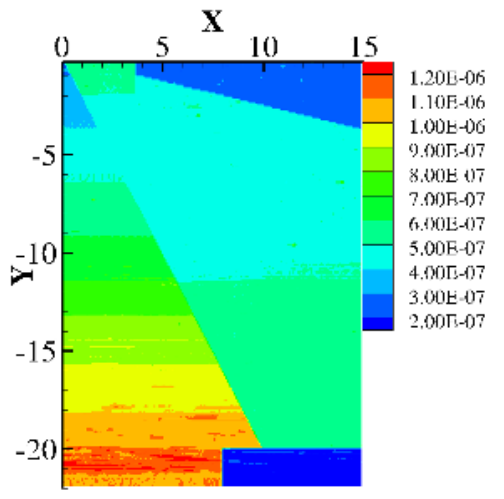

(a) Newman's method

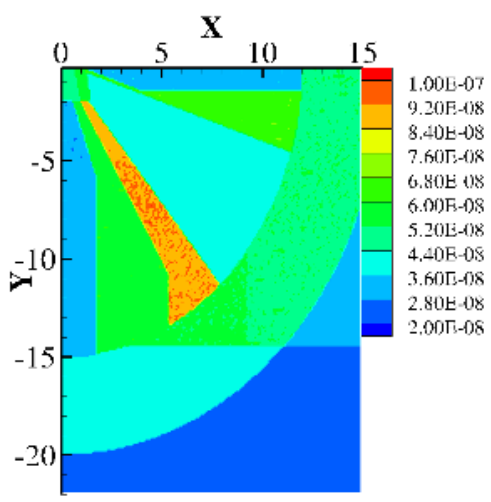

(c) Telste and Noblesse's method

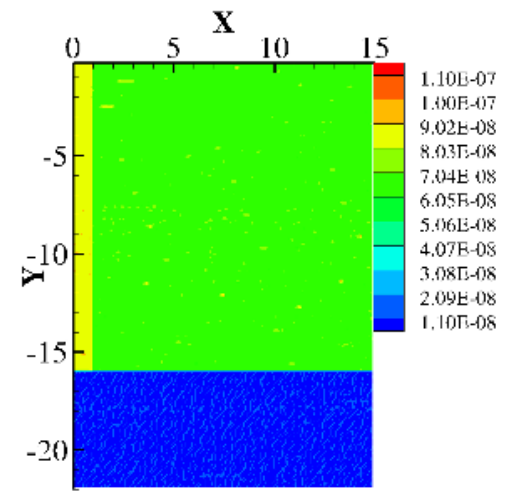

(b) Delhommeau's method

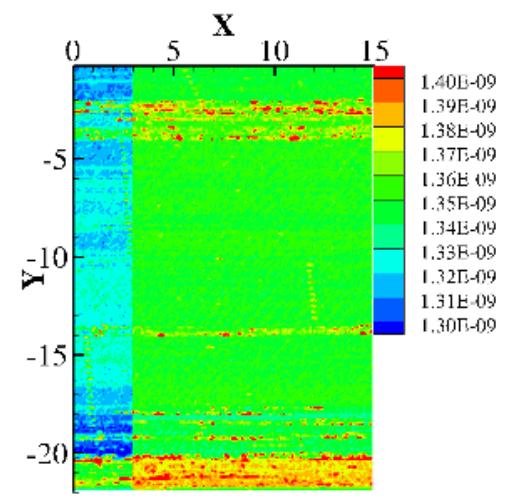

(d) Wu et al.'s method

Figure 7: Local computation time to calculate the Green function and its derivatives based on Newman's, Delhommeau's, Telste-Noblesse's, Wu et al's and direct integral method 
Table 6: Averaged computation time of Newman's algorithm

\begin{tabular}{|c|c|c|}
\hline \# of sub-domain & Corresponding $(X, Y)$ & Averaged computation time $(\mathrm{s})$ \\
\hline 1 & $(X>8,-Y>20)$ & $1.805 \times 10^{-7}$ \\
2 & $(-X / Y<0.5)$ & $8.439 \times 10^{-7}$ \\
3 & $(X>3.7,-X / Y>4)$ & $2.573 \times 10^{-7}$ \\
4 & $(0<X<3.7,0<-Y<2)$ & $5.265 \times 10^{-7}$ \\
5 & $(-X / Y \leq 4,-Y \leq 2)$ & $4.925 \times 10^{-7}$ \\
6 & $($ The rest part with $-Y>2)$ & $4.149 \times 10^{-7}$ \\
\hline
\end{tabular}

Table 7: Averaged computation time of Telste-Noblesse's algorithm

\begin{tabular}{|c|c|c|}
\hline \# of sub-domain & Corresponding $(X, Y)$ & Averaged computation time $(\mathrm{s})$ \\
\hline 1 & $\left(X^{2} / 12^{2}+Y^{2} / 15^{2} \geq 1\right)$ & $3.607 \times 10^{-8}$ \\
2 & $(0 \leq X \leq 1.2-0.15 Y,-2 \leq Y \leq 0)$ & $4.110 \times 10^{-8}$ \\
3 & $(X \leq \min (0.7 Y, 9.1))$ & $7.454 \times 10^{-8}$ \\
4 & $(X \geq-0.4 X)$ & $4.094 \times 10^{-8}$ \\
5 & (The rest part) & $6.699 \times 10^{-8}$ \\
\hline
\end{tabular}

\section{Conclusions}

In this paper, we reviewed the available mathematical expressions for the deep water free-surface Green function in frequency domain and four different numerical methods which were developed for its numerical evaluation. The four methods are the Newman's method, the Delhommeau's method, the TelsteNoblesse's method and the Wu et al.'s method. The computational time and accuracy with each method are compared. The Newman's method is the most accurate providing a 6 decimal accuracy. However, it is also the slowest. The Telste and Noblesse's method is an order of magnitude faster than the New- 
man's method, but it is slightly less accurate. The method by Delhommeau lie in-between the Newman and Teslte-Noblesse's method with respect to computational time but it is less accurate than the Telste-Noblesse's method. This makes this latter method preferrable to the Delhommeau's method. The Wu et al's method is also less accurate than the Newman and Teslte-Noblesse's method, but it is also the fastest method and the simplest with respect to numerical implementation (as they do not use different expressions depending on the position in the computational domain).

Finally, a limitation of this work is that only the case of deep water was considered despite the case of finite depth is also very important for practical applications. This is left for future work.

\section{Acknowledgements}

The first author is supported by the Chinese Scholarship Council(CSC).

\section{References}

[1] J. Newman, Algorithms for the free-surface Green function, Journal of engineering mathematics 19 (1) (1985) 57-67.

[2] G. Delhommeau, Amélioration des performances des codes de calcul de diffraction-radiation au premier ordre, in: Proc. Deuxièmes Journées de l'Hydrodynamique, Ecole Nationale Supérieure de Mécanique de Nantes, 1989, pp. 69-88.

[3] J. Telste, F. Noblesse, Numerical evaluation of the Green function of waterwave radiation and diffraction, Journal of Ship Research 30 (2) (1986) 6984.

[4] H. Wu, C. Zhang, Y. Zhu, W. Li, D. Wan, F. Noblesse, A global approximation to the Green function for diffraction radiation of water waves, European Journal of Mechanics-B/Fluids 65 (2017) 54-64. 
[5] C.-H. Lee, J. Newman, Computation of wave effects using the panel method, WIT Transactions on State-of-the-art in Science and Engineering 18.

[6] J. V. Wehausen, E. V. Laitone, Surface waves, in: Handbuch der Physik, Springer, 1960, pp. 446-778.

[7] F. Ursell, The periodic heaving motion of a half-immersed sphere: the analytic form of the velocity potential, long-wave asymptotics of the virtual mass coefficient, Tech. rep., Fluid Dynamics Branch, U.S. Office of Naval Research (1962).

[8] W. Kim, On the harmonic oscillations of a rigid body on a free surface, Journal of Fluid Mechanics 21 (3) (1965) 427-451.

[9] T. Havelock, Waves due to a floating sphere making periodic heaving oscillations, Proceedings of the Royal Society of London. Series A, Mathematical and Physical Sciences 231 (1955) 1-7.

[10] G. Hearn, Alternative methods of evaluating Green's function in threedimensional ship-wave problems, Journal of Ship Research 21 (2) (1977) 89-93.

[11] P. Guevel, J. Daubisse, G. Delhommeau, Oscillations des corps flottants soumis aux action de la houle, Bulletin de l'Association Technique Maritime et Aéronautique, 1978.

[12] D. Martin, Résolution numérique du problème linéarisé de la tenue à la mer, Bulletin de l'Association Technique Maritime et Aéronautique, 1980.

[13] F. Noblesse, On the theory of flow of regular water waves about a body., Tech. rep., Dept. of Ocean Eng., Massachusetts Inst. of Tech.(MIT) (1980).

[14] F. Noblesse, The Green function in the theory of radiation and diffraction of regular water waves by a body, Journal of Engineering Mathematics 16 (2) (1982) 137-169. 
[15] J. Newman, Double-precision evaluation of the oscillatory source potential, Journal of Ship Research 28 (3) (1984) 151-154.

[16] J. Newman, An expansion of the oscillatory source potential, Applied Ocean Research 6 (2) (1984) 116-117.

[17] C. Lee, J. Newman, WAMIT user manual, version 7.0, WAMIT, Inc.

[18] X. Chen, Evaluation de la fonction de Green du probleme de diffraction/radiation en profondeur d'eau finie-une nouvelle méthode rapide et précise, Actes des 4e Journées de l'Hydrodynamique, Nantes (France) (1993) 371-84.

[19] X. Chen, Free surface Green function and its approximation by polynomial series, in: Bureau Veritas' research report No 641 DTO/XC, Bureau Veritas France, 1991.

[20] Hydrostar for experts user manual, Bureau Veritas, 2016.

[21] R. Wang, The numerical approach of three dimensional free-surface Green function and its derivatives, Journal of Hydrodynamics 3 (1992) 277-286.

[22] A. Babarit, G. Delhommeau, Theoretical and numerical aspects of the open source BEM solver NEMOH, in: 11th European Wave and Tidal Energy Conference (EWTEC2015), 2015.

[23] M. Ba, B. Ponizi, F. Noblesse, Calculation of the Green function of waterwave diffraction and radiation, in: The 2nd International Offshore and Polar Engineering Conference, International Society of Offshore and Polar Engineers, 1992.

[24] B. Ponizy, F. Noblesse, M. Ba, M. Guilbaud, Numerical evaluation of freesurface Green functions, Journal of Ship Research 38 (2) (1994) 193-202.

[25] M. A. Peter, M. H. Meylan, The eigenfunction expansion of the infinite depth free surface Green function in three dimensions, Wave Motion 40 (1) (2004) 1-11. 
[26] J. D'elía, L. Battaglia, M. Storti, A semi-analytical computation of the Kelvin kernel for potential flows with a free surface, Computational \& Applied Mathematics 30 (2) (2011) 267-287.

[27] B. Borgarino, A. Babarit, F. P, Extension of free-surface Green's function multipole expansion for infinite water dapth case, International Journal of Offshore and Polar Engineering 21 (3) (2011) 161-168.

[28] A. Clément, A second order ordinary differential equation for the frequency domain Green function, in: 28th International Workshop on Water Waves and Floating Bodies, 2013.

[29] H. Liang, H. Wu, F. Noblesse, Validation of a global approximation for wave diffraction-radiation in deep water, Applied Ocean Research 74 (2018) $80-86$.

${ }_{615}$ [30] T. Havelock, The damping of the heaving and pitching motion of a ship, Philosophical Magazine 33 (7) (1942) 666-673.

[31] M. Haskind, On wave motion of a heavy fluid, Prikl. Mat. Mekh 18 (1954) $15-26$.

[32] R. Yeung, A singularity method for free-surface flow problems with an oscillating body, Tech. rep., University of California, College of Engineering, The Office of Naval Research, Report No. NA 73-6, Berkeley, USA (1974).

[33] M. Abramowitz, I. A. Stegun, Handbook of mathematical functions with formulas, graphs, and mathematical tables, Government Printing Office, Washington and Dover, New York, 1964.

${ }_{625}$ [34] S. Liapis, Numerical methods for water-wave radiation problems, International Journal for Numerical Methods in Fluids 15 (1) (1992) 83-97.

[35] N. Basu, On double Chebyshev series approximation, SIAM Journal on Numerical Analysis 10 (3) (1973) 496-505. 
[36] G. Delhommeau, Les problèmes de diffraction-radiation et de résistance des vagues.pdf (1988).

[37] J. L. Hess, D. C. Wilcox, Progress in the solution of the problem of a threedimensional body oscillating in the presence of a free surface, Tech. Rep. DAC 67647, McDonnell Douglas Corp., Long Beach, California (1969).

[38] J. Newman, The approximation of free-surface Green functions, in: P. Martin, G. Wickham (Eds.), Wave asymptotics, Cambridge University Press Cambridge, UK, 1992.

[39] J. Newman, Approximations for the Bessel and Struve functions, Mathematics of Computation 43 (168) (1984) 551-556. 